\title{
Simulating House Cooling Methods to Decrease Energy Consumption by Creating Awareness and Attitude Change
}

\author{
Clinton Arendt ${ }^{1}$, Trina Myers ${ }^{1,2}$ and Jarrod Trevathan ${ }^{3}$ \\ ${ }^{1}$ Discipline of IT, School of Business, James Cook University, Queensland, Australia \\ ${ }^{2}$ Centre for Tropical Biodiversity and Climate Change, James Cook University, \\ Queensland, Australia \\ ${ }^{3}$ School of Information and Communication Technology, Griffith University, Australia \\ ${ }^{1,2}$ (Clinton. Arendt; Trina. Myers)@jcu.edu.au \\ 3.trevathan@griffith.edu.au \\ Abstract
}

\begin{abstract}
Efficient cooling methods are necessary when living in a topical climate where high temperatures and high humidity are normal. Air-conditioning units, which are expensive to run and maintain are standard in a majority of houses in Australia's tropic zone due to the design of both historic and modern housing not being fully sutted to a tropical region. Significantly, the cost of energy consumption continues tone the There are simple and inexpensive modifications a homeowner can do naturalt cool the house and preserve energy, such as, painting roofs white, planting screening vegetation and installing shades externally to cover windows, etc. However, mano of these alternative techniques are not common knowledge and are not presented to the public in ways that can inform and potentially change behaviour. This paper pesents a housing simulation prototype that applies gamification methods to build awareness on ways to reduce cooling power consumption. An interactive guided persusion methodology is used to educate homeowners about these alternative rechniques so they may choose natural cooling methods instead of relying on electrical cooling appliances. The results of the user testing show the simulation design did encourgge awareness and motivation to make tangible adaptations to homes.
\end{abstract}

Keywords. Smart homes, Simulation, gamification, persuasive computing

\section{Introduction}

The tropics of the world have the combination of high temperature and humidity, which often reach levels that are unbearable to humans. To maintain a comfortable living standard in tropical regions, artificial environmental control devices are used, such as air-cooling or airconditioning units. Recently, there has been a rapid growth in the use of air conditioning system in densely populated tropical areas in Australia, which is contributing to the pressure on the electrical grid infrastructure [2,3]. The continual increase in air conditioning units and public overuse puts excessive pressure on the electricity grid, which has contributed to the increase in electricity costs. New properties that are erected in northern Australia are still predominantly driven by cost of building and not cooling efficiency $[4,5]$. Although housing design and construction has progressed significantly in the past fifty years, modern housing developments are designed for air-conditioning and not alternative home-cooling methods. Research on effective home-cooling techniques has found numerous methods to reduce the temperature and humidity in the home without the use of air-conditioning [5, 6]. For example, a lower internal absorption of heat can be achieved by applying white reflective paint to a 
structures roof and strategic interior design of rooms can maximise airflow and/or minimise heat from the afternoon sun. However, these techniques are not common knowledge; there is a disconnection between the research knowledge and public awareness.

Although current education initiatives are in place to build this awareness, they are predominantly informational pages on council web sites. There are limited applications designed for simulating single home environments that are easy to use by the public and focus on energy awareness in the home. Public education on home-cooling would present information about ways to modify homes effectively to make them more efficient and allow some users to extrapolate the changes that are needed to improve their own homes. However, this information is not in aggregated form for easy consumption by a wider general audience.

Gamification techniques and simulation can be applied to present this information in an engaging and interactive manner. Gamification is a framework for enhancing user interactivity and increasing user motivation and engagement with a system and is often ased in educational tools [7]. Gamification techniques provide consistent feedback and activity based on the actions of the user and delivers final state information so the user may understand and compare the effect of their decisions [8]. Current housing simulation software comprise mainly of applications that require steep learning curyes to operate and extensive knowledge of commercial building. The currently available simulation sof tware packages are very complex and take in large amounts of data input, such as structural design information and influence variables. From these inputs, the simulations output vast amounts of data that requires dissection and correlation with other simulations.

This paper presents a three-dimensional (3D) simulation software solution that guides and informs the user towards effective cooling methods for their particular house design. The prototype implementation uses gamification techniques aimed at a user persona of a homeowner to educate and inform about building materials and strategic landscaping that can naturally reduce heat within the home. The 3D simulation lets the user modify their home for more effective cooling through determinants such as roof and external wall paint colour and reflectivity, insulation, roøm positiontng, shade and awnings, etc. The software's user interface shows the household energy use is decreased with each additional natural cooling technique.

The prototype was tested by sample of the end-user to determine its effectiveness for education and behavioural change about responsible energy use and cost effective climate control through house design The results show the simulation game-based design did act as a catalyst to encourage awareness and motivation to make tangible home-cooling adaptations to homes.

This paper is structured as follows: Section 2 presents background information on homecooling methods and the technologies that can bridge the knowledge gap. Section 3 explains the design methodology of the simulation tool and Section 4 describes the prototype design. Section 5 presents the user analysis and discussion and finally, a brief summary and conclusion is provided.

\section{Background}

The use of electricity is growing due to the increase in the global population and its dependency on electronic appliances [3]. New appliances are continually being introduced to the market and their usage steadily increases the use of electricity. Electricity grids have had to expand and consume more fuels to keep up to the demand from the proliferation of modern appliances. Included in the massive growth in electrical items are home environment 
appliances such as heating and cooling systems. This paper concentrates on home-cooling methods in the tropic zone so the focus is on air-cooling and -conditioning appliances.

Although these cooling appliances have improved in efficiency since their initial introduction to the market, they are still high-energy consumers [9-11]. Heating, Ventilation and AirConditioning (HVAC) electricity consumption typically accounts for around $40 \%$ of total building electricity consumption and $70 \%$ of base building (i.e., proprietor) consumption. HVAC dominates peak electricity demand, so improving its efficiency can reduce peak demand electricity charges [9]. Notably, high performing buildings are now getting better returns as tenants prefer to occupy sustainable buildings.

\subsection{Natural Home-cooling Methods}

Heat transference is possible via radiation, conduction and convection. Radiation occurs when a heat source warms a surface such as the sun heating the interior by shining on the building's roof. Conduction occurs when heat is transferred through solid objects such as from the external wall to the internal plaster. Convection happens when hea is carried by circulation of gases and liquids, such as when hot air rises drawing cooler air from below.

Homes can be modified to lessen their demand for electrieity and environmental impact using techniques for cost effective natural cooling. Methods of internal home temperature improvement in the tropics include: reflective roof and wall paints, afternoon sun wall shading with either vegetation or physical shading, wall materials, insulation and window tinting $[5,6,12]$. Some methods should be implemented dưring the design and initial building of the home (e.g., materials, wall insulation, location based on afternoon sun, etc.,). However, the focus of this paper is on the post constryction modilications and methods of which many are inexpensive and simple to implement. Some of these methods include:

-Landscaping - which can provide shade to the garden areas and to as many of the external walls and as much of the root of a home as possible. For example, a tree to shade the living room would make a considerable difference to internal heat levels by minimizing heat transference by conduction;

- Roof and wall colour - Roofs and external walls painted with light colours reflect heat, which can counter heat transference by radiation and conduction;

-Shading Eaves and awnings are essential for houses in tropical climates, and combined with appropriately located outdoor living areas assist in shading main windows and exposed walls. Eaves and awnings work well in the tropic zones where the summer sun is at a higher angle;

- Insulation-Reduces the amount of heat being transferred into the home. It works by resisting the amount of conducted and convected heat flow between the hotter air in the roo-space or wall-space and the cooler air inside the home;

- Crrculation - Poorly ventilated areas such as roof-spaces get hot. To counter heat ransference by convection techniques include the use of whirly-birds and vents to anow the heat to circulate out;

- Outdoor living spaces - Maximise the use of outdoor areas through correct location, integration with indoor environments and creating 'transitional spaces' into breezeways; and

- Grass and water versus uncovered paved areas - Minimise the amount of hard surfaces that radiate heat such as driveways or concrete footpaths [5, 6].

These simple modifications can cumulatively lessen the electricity demand and consumption by decreasing the use of air-conditioning. However, there is limited public 
awareness of these techniques. The aim of this project was to create this awareness via simulation and gamification techniques.

\subsection{Current Housing Simulation Software}

Current housing simulation software packages comprise mainly of applications that require steep learning curves to operate and extensive knowledge of commercial building; few are designed for simulating single home environments. These packages take in large amounts of data input, such as structural design information and influence variables, to output extensive amounts of data that requires dissection and collaboration with other simulations. Due to this complexity, current simulation software applications are mostly inoperable by the public.

Notably, the current housing simulation software often only simulates commercial buildings. In addition, the environmental variables, such as local weather data, etc., and the building standards pertain to the location of the software developers, which are predominantly North American, because these variables are often taken from static sources [13] Most opensource simulation software packages require extensive knowledge of/many aspects of building design and environmental data inputs. A solid understanding of the data used in house-design is required to navigate these open-source mterfaces and dat input methods, which are often unintuitive and lacking in documentation

eQUEST [14] and Home Energy Efficient Design (HEED) [13] are two popular simulation software packages that allow simplistic user interaction. eQUEST is a freeware commercial building simulator and was built on the software engine DOE-2 which was developed by the Lawrence Berkeley National Laboratory [14 eQUEST adds design wizards and graphics to the DOE-2 engine to make the design flow simpler. Even with the reduction in complexity, the user is required to have knowledge of construction and house design data to operate the eQUEST system. HEED is a freewatre simulation software package developed by the University of California to target Californiân ratepayers and homeowners [15]. HEED was designed to simulate a single house enviroment to see how much energy, money and carbon a Californian homeowner can save by making various designs or remodelling changes to the home. The HEED system was classified as an "information only" program focused in a specific geographic region.

\subsection{Early Studies that used DOE-2 Simulation Software}

DOE-2.1C software is the predecessor of eQUEST and was used to produce simulations of vegetation potentials on summer cooling load reduction to analyse cost and determine energy use in buildings [12]. Using the DOE-2.1C simulation program, Huang et al., [12] calculated net reductions in air-conditioning in three locations; Sacramento California, Phoenix Arizona and Lake Charles Louisiana, due to trees and other vegetation. The simulation results indicated that buildings shaded by vegetation, taking into account the Evapotranspiration (ET) properties, considerably decreased peak-power consumption. In fact, the peak-power consumption was reduced by $34 \%$ in Sacramento USA, $18 \%$ in Phoenix USA and $22 \%$ in Lake Charles USA with $30 \%$ vegetation shading and ET.

DOE-2.2, and its expansion eQUEST, are the successors of the DOE-2.1C and DOE-2.1E engine [16]. eQUEST uses an enhanced Graphic User Interface (GUI) technology, which was initially developed for the commercial version PowerDOE. The GUI was designed to use Computer Aided Design (CAD) file import for building geometry and graphical displays of HVAC equipment layout. eQUEST and its derivatives, DOE-2.2 and lower, perform well but the input and output data requires expert knowledge and configuration, which is not suitable to a wider public audience. 


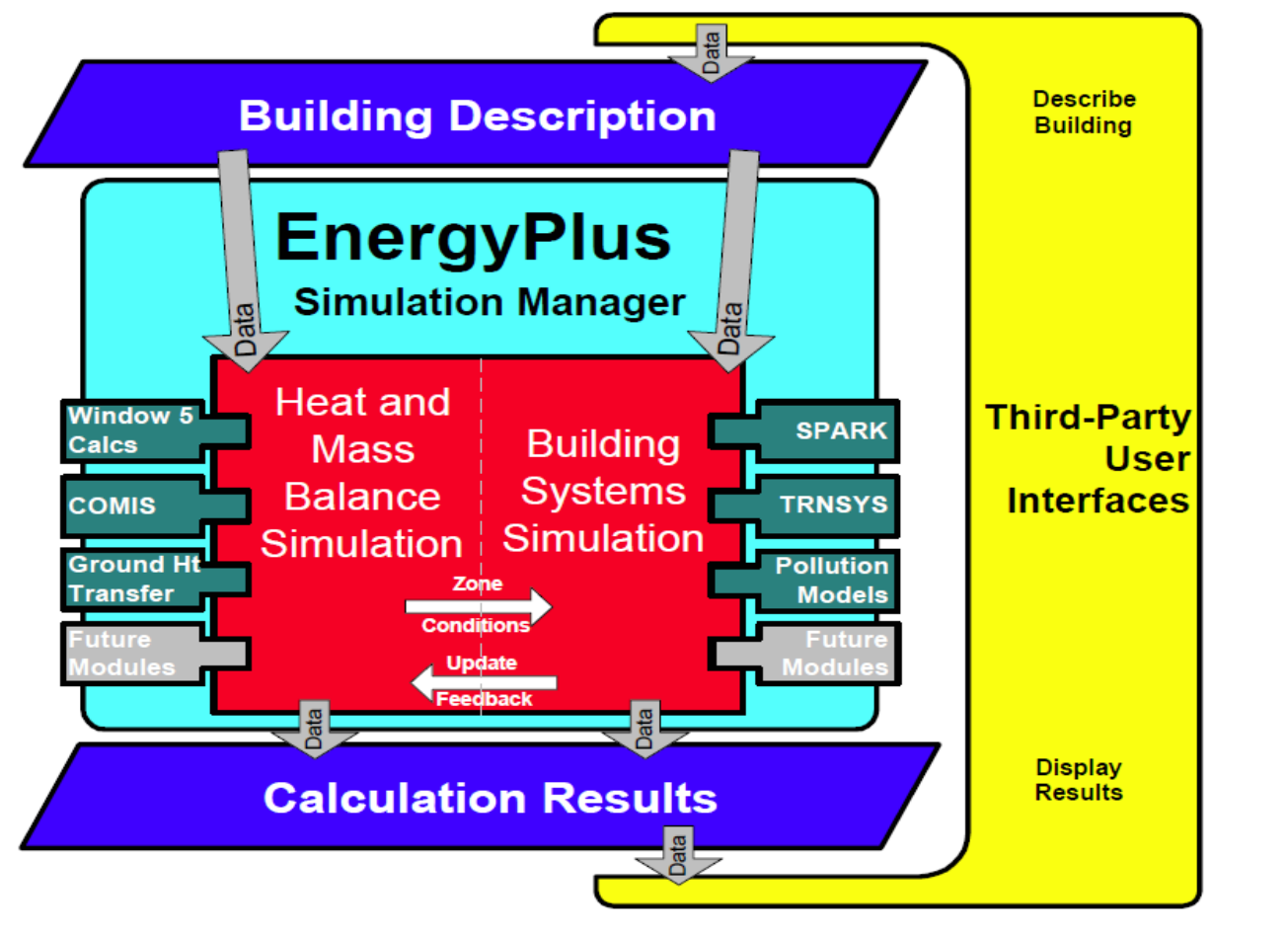

Figure 1. The EnergyPlus Structure [1]

EnergyPlus was based on the most popular features of BLAST and DOE-2.2 [1]. It was developed by the U.S. Army Construction Engineering Research Laboratories, University of Illinois, Lawrence Berkley National Laboratory, Oklahoma State University, GARD Analytics and the US Department of Energy (DOE) [17]. EnergyPlus is still initially a command line program and like DOE 2 and BLAST, it relies on third parties to design and develop user interfaces.

Most simulation software constructs their results by passing data through multiple layers of calculations Figure 1. Energy Plus developers designed their system with the ability to activate plug-ins throughout the calculation process to create accurate data. Figure 1 shows how calculation results are formulated where the user describes the building they wish to create through third-parry user interfaces. The building description data is passed to simulation manager where the heat and mass balance and building systems simulations perform the specified range of calculations. The data is then collated at the calculation results level and supplied to the user interface for viewing.

The lis of simulation situations that can be applied with EnergyPlus is introduced in the software documentation [17]. Some of the situations that are not present in DOE-2 are:

-Anisotropic sky modelling, which improves calculations of diffuse solar on tilted surfaces.

-Transient heat conduction, which allows conduction of heat through building materials.

-Day lighting controls, allowing calculations of luminance, glare simulation and control, luminaire controls and the effects of reduced artificial lighting on heating and cooling. 


\subsection{Comparison of a Majority of Available Building Simulation Software}

Current building simulation software is not aimed for general use, they are predominantly research and development tools for efficient building designs. Crawley et al., [13] presented an comparison paper examining the capabilities of twenty different building simulation software packages. The comparison examined how well each building simulation package performed the desired function and included the following different usability functions:

$\mathbf{X}$ - Features or capabilities that are available and in common use;

$\mathbf{P}$ - Features or capabilities that are partially implemented;

O - Optional features or capabilities;

$\mathbf{R}$ - Optional features or capabilities that are for research use;

$\mathbf{E}$ - Features or capabilities that require domain expertise; and

I - Features or capabilities that are difficult to obtain input.

The degree of user confidence in the simulation is important [13]. The simalations provide the results via specific calculations, which must have trust, are accurate. The calculations in the open source software could be easily examined whereas inproprietary software, the user must trust documentation [13]. Based on the comparisons, the better simulation systems avoid over complicating the calculations. The system presented in this paper applies methods to simulate simple environmental variables without highly complex mathematical calculations.

\subsection{Human Computer Interaction}

Human computer interaction (HCI) is the study of the interaction between humans and computers. The interaction is usually examined by monitoring usage and recording feedback on designs and functionality and adjusting technologies accordingly. The following sections discuss design methods in human computer interaction that can be applied to educate and change attitudes.

2.5.1. Computers as Tools, Media and Social Actors: Computing technologies can be used as tools, medium or as social actor to persuade users [18, 19]. As a tool, computer technology can increase user capability For example, user goals are easier to achieve, their productivity jncreased through set processes and they can perform calculations that would otherwise be impossible with conventional tools. As a medium, computers can provide experience through exploration of cause-and-effect relationships, motivation through vicarious occurrences and behaviour rehearsal. As social actors, the computer can play a social role by rewarding users with positive feedback, modelling target behaviour and providing shared community support. These persuasive techniques can be applied in combination to change behaviour.

2.5.2. Tailored User Experience: To properly develop an educational tool for a vast and varying target audience the application should fulfil most of these roles. Although this presents development difficulties of presenting too much information or guidance, information that should be adjustable for the user [20]. Allowing users to view the information that they require and avoiding information that they do not is a key factor in producing an easy to use cross-user compatible system.

2.5.3. Guided Persuasion: Guided persuasion is another persuasive technology method, referred to as tunnelling, that presents the user with a step-by-step process from which they cannot defer $[18,21]$. The tunnelling method removes user direction and replaces it with a 
forced track that can only be traversed lineally (i.e., forward or backward). Users are often forced to process all of the information given for them to progress as they are not allowed to make their own choices. Tunnelling is a useful technique when a single goal is to be achieved, but it does makes arriving at conclusions from their experience difficult for users.

2.5.4. Suggestion Technology: Suggestion technology is the technique of analysing the user position and suggesting various ways on how to continue. Different variables must be taken into consideration to make effective suggestions, such as user disposition, the need of suggestion, the suggestion location and timing. This technique is often used in conjunction with tunnelling to present a more enjoyable experience and increase the user's ability to flow fluidly through applications.

\section{Design Methodology}

This project aimed to develop software that was usable by the public so 1 operability, simplicity, appeal and output were design focuses. The goal was to ensure users had the ability to easily create a simplistic re-creation of their own home so the could be guided towards modifying the home for more effective cooling.

Usability can be ascertained through measures of effectiveness, efficiency, and satisfaction quality factors [22]. To evaluate these factors, knowledge of the target audience and their abilities to operate and interact with specifio applications is essential. This simulation software was designed for a general public target audience with a demographic indicative of a homeowner (e.g., age from 25 years, mid to high income, etc.,). Because the end user's computer literacy may vary from novicelogexpert the user interface needed to be simplistic with no ambiguous elements and lollover effects on elements that require instant explanations. The software must cater for the user type that is most unfamiliar with using simulation products so it should provide clues to the user to guide them through the application. The software user interface sh ould be easily visible, without clutter and operate according to explained operation [22].

Operability refers to the compatibility of software and hardware platforms. Software developed for a public audience should take consideration of the current state of public domain applicatiôns, installation issues, bandwidth and resource demands to provide a solution that is accessible to the majority of users [23]. The software was designed for interoperability to maintain user interface flow.

Software designed o ennance the user experience must aim for consistent simplicity and appeal without oven simplification. The software should show all available outputs without over loading the usen with unnecessary data and unusual presentation [24]. The appeal of the software developed includes consistently maintaining the colour scheme, icon style, font theme and general design.

The soltware output should be information the user requires and not information that is foreed upon them [24]. Output data and information can often overload users and can lead to proble ns of over simplification. To combat over simplification, the software should be able to represent the data in useful, easily understandable forms so the user can interpolate to find the information they are seeking.

The project described in this paper developed software that allowed users to make modifications to their house with simplistic controls in a 3D environment. The house design variables consisted of building materials, roofing style and colour, window positioning and air-conditioning nodes. The environmental variables included temperature, humidity and 
wind flow to display high temperature zones and wind flow paths. Other outputs include energy/cost to cool particular rooms, temperature and humidity graphs.

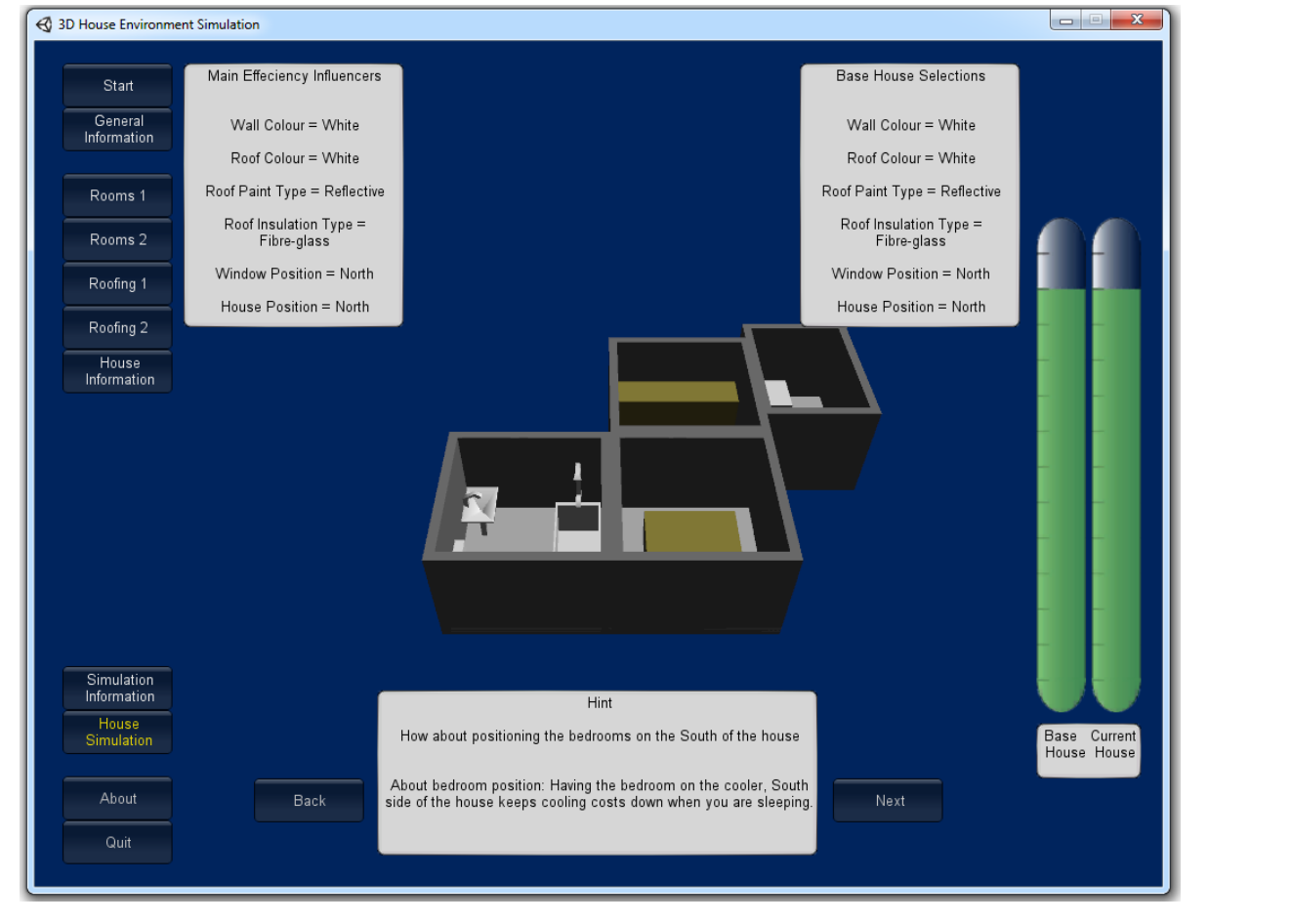

Figure 2. Prototypes Generic Hôuse yvith Four Rooms - from Top Left: Bathroom, Kitchen, Bedroom and Laundry

\section{Prototype Design}

The HCI methods used in the implementation included guided persuasion and suggestion technology. These methods were adapted to make an educational and potentially attitude changing application. Users learn the different natural cooling methods of house design through step-by-step guided persuasion and hints are interwoven with suggestion technology once a house has been bulit.

A 3D House simulation prototype was developed to structure the menus and guides adequately so they would help users with the many internal elements and determinants of house development and cooling efficiency. Unity3d [25] was used as the game development framework. Unity $3 \mathrm{~d}$ is a powerful rendering engine fully integrated with intuitive tools and rapid Orknlows to create interactive 3D content. Unity supports multiplatform publishing, which enables output to different operating systems, devices and browsers via a built-in web player. Due to the ease of quick application output, debugging and modification throughout the development and testing phases were simple and efficient.

\subsection{The Simulation Software Rooms, Menus and Design}

A "generic" home was developed, which consisted of a kitchen, laundry, bathroom and bedroom to reduce complexity. These four rooms may not be present in all houses but they offer a good example of what can be expected with most modern homes. These "generic" 
rooms present elements that are to be modelled, such as wall insulation, window position, etc., which are automatically applied to all simulations without user interaction. Figure 2 shows a simulation screenshot of these generic rooms.

The menu consists of the options a user may choose to modify each room. Radio buttons were used for the selection method for options that have only one answer from a finite number of variables (Figure 3). For example, the user is given three options to determine the number of bedrooms (i.e., one, two or three bedrooms). The different modification options for

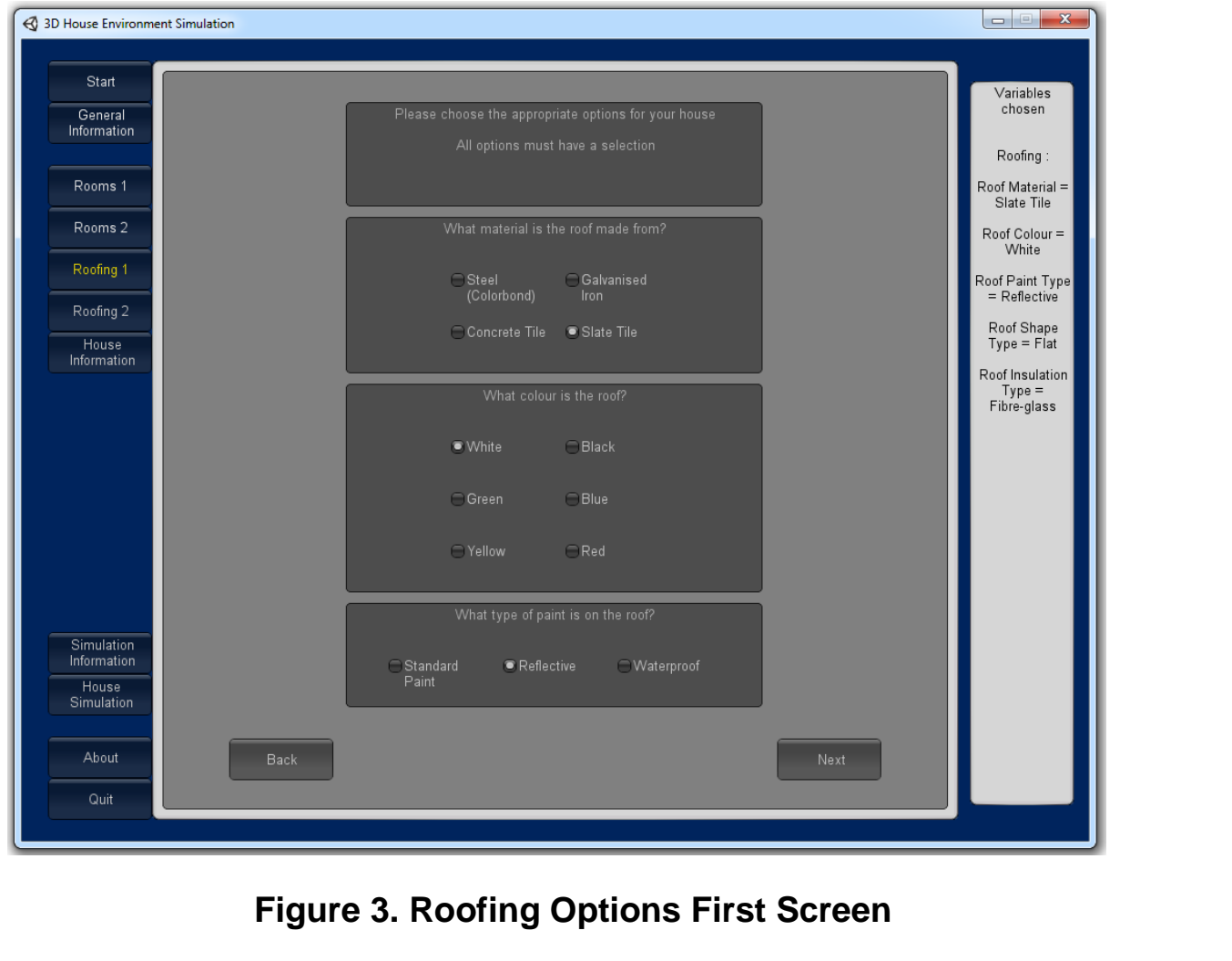

the house were separated inder section tabs to prevent cognitive overload where the user is faced with too many decisions. These tabs also allows for easier traversal through the entire simulation as the user ar visibly see their progress. The menus allows for easy setting and integration of the variables for cooling efficiency.

\section{User Andlysis and Discussion}

An end user analysis was conducted to collect data to gauge the effectiveness of the prototype The user testing was performed with a sample size of thirteen in a control environment at the James Cook University campus to maintain consistent participant conditions. The demographic criteria for the key persona groups included groups for age, home ownership status and computer literacy. The preferred participants were to be current home owners because the trial was to examine the likelihood they would make modifications to their homes after taking part in the simulation.

Qualitative and quantitative data were gathered through individual interviews and a questionnaire given during user testing. The interviews were conducted individually prior to user testing to assess the participant's views and motivations toward conserving energy in 
their homes. Interviews were also conducted after the user test to gauge attitude change through anecdotal evidence. The questionnaire given during the testing process examined the users experience or feeling via a five point. The Likert scale in this questionnaire is presented as strongly agree to strongly disagree. Further qualitative data was gathered through a set of open-ended questions that were part of the user testing survey. The participants were asked to reflect on their feelings and experiences about the simulation.

The participants were given identical situations to work through the simulation while completing the questionnaire. The sample of the population included differing age groups to examine both varied home ownership stages and familiarity to the digital medium (Table 1). Grouping participants by age could determine assumed levels of computer literacy. That is, the assumption is that people aged below fifty have had a higher exposure to computers and digital equipment then people over fifty. The effects of user experience were gauged by both questionnaire and observation. Notably, the participants aged from 40 to 49 found the prototype easier to use yet recorded the lowest daily usage of computers, whichincicates the prototype was user friendly.

Table 1. The Demographics and Characteristics of Participants as the Sample Population

\begin{tabular}{|c|c|c|c|c|}
\hline Age Group & Quantity & $\begin{array}{l}\text { Computer } \\
\text { literacy level } \\
\text { (mean) }\end{array}$ & $\begin{array}{l}\text { Aware of cooling efficiencies } \\
\text { deficiencies within their own } \\
\text { home (mean) }\end{array}$ & $\begin{array}{l}\text { Found the prototype } \\
\text { enabled them to create } \\
\text { the sample house } \\
\text { quickly (mean) }\end{array}$ \\
\hline $20-29$ & 3 & Medium high & & High \\
\hline $30-39$ & 4 & Mediur & & High \\
\hline $40-49$ & 3 & Mediun & & Very High \\
\hline $50-59$ & 2 & High & & High \\
\hline $60-80$ & 1 & Yery High & high & Medium \\
\hline
\end{tabular}

*Computer usage among allage groups was high with only one participant recorded with a low computer literaçy.

\subsection{User Testing Results - Quantitative}

The results of questions pertaining to awareness and changing attitude are presented in Figure 4. The results Show the participants initially had a low awareness of the different cooling methods to cool the house naturally and the awareness increased as they worked through the simulation.

The results further show that the use of this kind of simulation software would act as a catalyst to changing behaviour. The questions pertaining to the educational and informative elements (i.e, "Did the software make you more aware of the possibilities to improve cooling efficiency within your home?" and "Did the software make you more aware of your home's cooling deficiencies?") show positive responses by the majority of participants. 


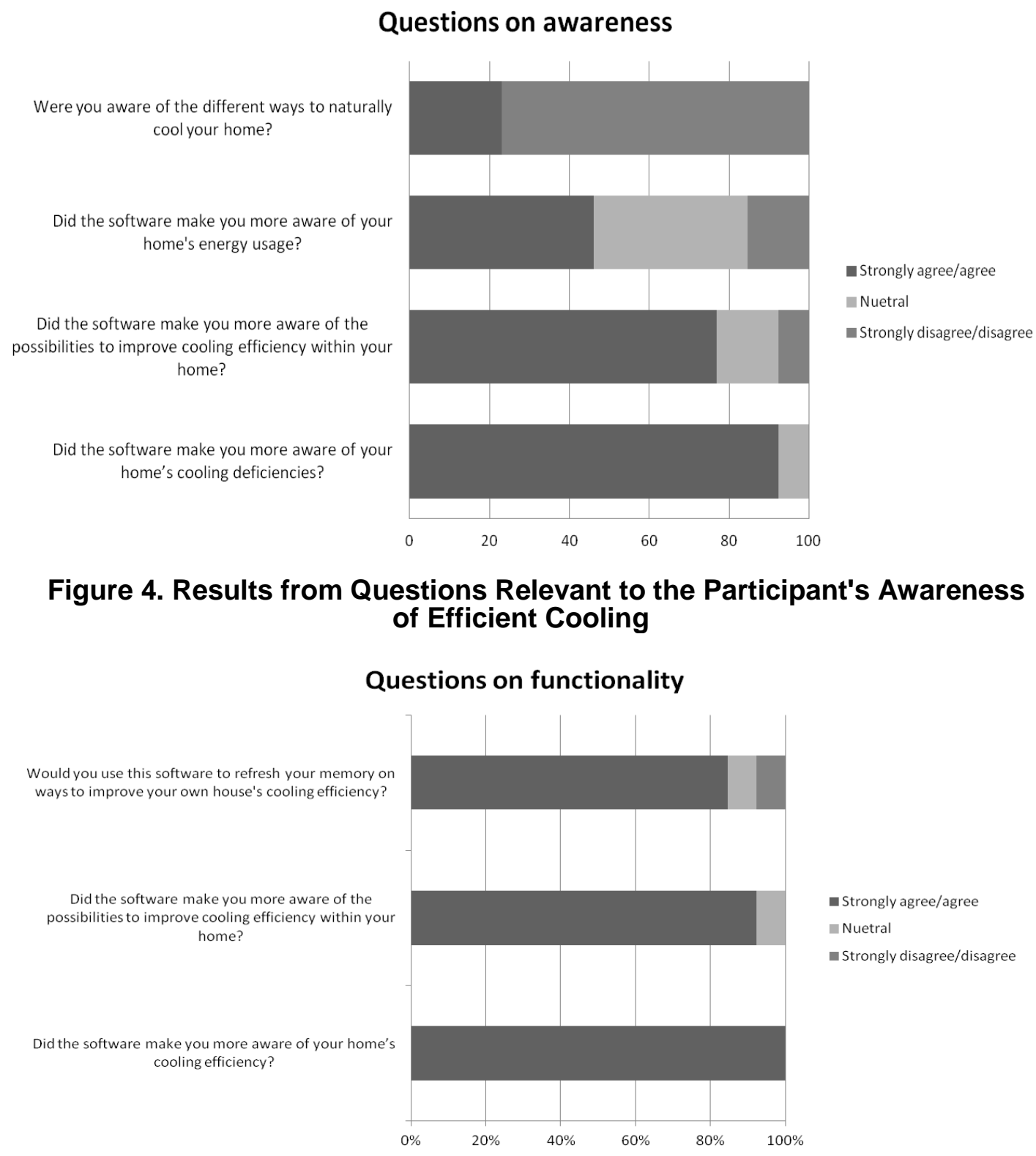

Figure 5. Questions Relevant to the Functionality of the Prototype Simulation Software

Questions were asked about the interface functionality and the usability of the software itself(Figure 5). The survey questions that aimed to determine whether a software simulation tool can be designed to cater to a diverse range of user profiles and computer experience to ensure a wider maximum reach of audience. The positive participant response is shown in Figure 5, which confirms the research question. Not only did all users complete the simulation without difficulty they also found the method of having to repeatedly view settings to make changes made them more aware of the individual elements of efficient home-cooling. 


\subsection{User Testing Results - Qualitative}

\section{Table 2. Anecdotal Evidence from the Interviews (Before and After Testing) of the Change in Perspective on Natural Home-Cooling Methods Versus Air Conditioning}

\begin{tabular}{|c|c|}
\hline Theme & Responses \\
\hline \multirow{2}{*}{$\begin{array}{l}\text { Views and motivations } \\
\text { toward conserving energy }\end{array}$} & "I would love to bring my electricity bill down!" (group 20-29) \\
\hline & $\begin{array}{l}\text { "I have all the energy saving lights installed and am very conscious of using } \\
\text { electricity, but during summer I need the air conditioning!" (group } 40-49 \text { ) }\end{array}$ \\
\hline \multirow[t]{3}{*}{ Awareness } & $\begin{array}{l}\text { "I had no idea the colour of the roof would make any difference to the temperature } \\
\text { inside my home" (group 40-49) }\end{array}$ \\
\hline & $\begin{array}{l}\text { "I know vegetation in front of my windows will bring down the temperature but was } \\
\text { not aware it made any difference to air conditioning energy use" (group } 30-39)\end{array}$ \\
\hline & $\begin{array}{l}\text { "Made me think more about the paint and window positioning then before", "I now } \\
\text { know how to cool my home via electronics, pain and positioning. "(group 20-30) }\end{array}$ \\
\hline \multirow[t]{3}{*}{ Change indicators } & "I'm going to call the roof seal people to paint my \\
\hline & $\begin{array}{l}\text { "How simple is that? Just plant a shrub in front of that window on my western side. . } \\
. \text { " (group } 20 \text { - 29) }\end{array}$ \\
\hline & $\begin{array}{l}\text { "I am going to plant more trees!" (group } 20 \text { - 29) } \\
\text { "Moving bedrooms helped, reflective paint andinsulation made a large difference" } \\
\text { (group } 30-39 \text { ) }\end{array}$ \\
\hline
\end{tabular}

The written responses by the users and discussions from both pre and post interviews were analysed to determine behavioural change indicators Judgments based on singular comments do provide an insight into how users percelve their experience. The responses were categorized into the following three themes: 1) Views and motivations toward conserving energy; 2) awareness and 3) indications of behavioural change (Table 2). The anecdotal evidence from the interviews and the openended survey questions show distinct implications for behavioural change in fact, there were many instances where users verbally indicated they would be able to make accurate decisions on the simple cooling efficiency changes on their own home afterusing the prototype.

Notably, there were comments can help improve the interface in future work. Although the user testing showed participants found the prototype easy to navigate and use, many users found the first screen (the start screen) to be ambiguous as to what action to perform. Some users found the representation of rooms hard to distinguish from one another and suggested appropriate labelling would improve the experience.

\section{Conclusion}

Thi project explored how persuasive computing techniques could be applied to educate and change attitude about housing design for cooling efficiency. This project built a simulation software application to aggregate natural home-cooling techniques using persuasive computing techniques to educate homeowners on simple actions they can perform to save energy.

The goal was to reduce the demand on electricity by educating homeowners about alternative techniques used to cool a house in tropical regions. The target audience were people that could make decisions about modifications and renovations to housing properties, which include homeowners and some rental tenants. 
This project is significant to educating people about sustainable living by changing attitudes towards the use of air-conditioning. The study concluded simulation and gamification applications can create an awareness that may positively persuade people to change behaviours. The project showed that 3D house-environment simulation software can be a catalyst for changing attitudes and/or behaviour about efficient home-cooling. The study showed these kinds of applications can inform and educate users about simple changes that can improve the efficiency of cooling their homes and therefore reduce reliance on airconditioning.

Future work includes developing the system further to include other climate zones. For example, if the system was extended to incorporate the southern temperate zone, which is a colder climate, the suggested modifications would include techniques to retain the heat inside the house.

\section{Acknowledgements}

The authors wish to thank the Townsville City Council's Sutstainability Education Centre for their guidance and expertise and Professor Ian Atkinson, Michael Cook and Dr Tim Marsh from James Cook University for their insight and advice.

\section{References}

[1] D. B. Crawley, L. K. Lawrie, F. C. Winkelmann, W. Buhl, Y. J. Huang, C. O. Pedersen, R. K. Strand, R. J. Liesen, D. E. Fisher and M. J. Witte, "EnergyPlus: creating a new-generation building energy simulation program", Energy and Buildings, vol. 33, no, 4, (2001).

[2] A. Auliciems and R. Dedear, "Air conditioning in a tropical climate: Impacts upon European residents in Darwin, Australia", International Journal of Biometeorol gy, vol. 30, no. 3, (1986).

[3] Australian Department of the Environment Water Heritage and the Arts, Energy use in the Australian residential sector 1986-2020 [electronic resource] Dept, of the Environment, Water, Heritage and the Arts, Canberra, A.C.T, (2008).

[4] A. Aitken and S. Losee, "To A/C or not, A/C? That is the question. Climate control in the mixed subtropical climate of SE Queensland", presented at the Subtropical Cities 2006 Conference, Brisbane, Australia, (2006).

[5] Department of Local Government and Planning, "Design guide for 6-star energy equivalence housing", Department of Housing and Public Norks, 2011).

[6] Townsville City Council, Sustainable Housing [WWW site]. Available: http://www.townsville.qld go/au/resident/planning/sustainable/Pages/default.aspx, (2013) June.

[7] A. Domíngutez, J. Saenz-de-Navarrete, L. de-Marcos, L. Fernández-Sanz, C. Pagés and J.-J. MartínezHerráiz, "Gamifying learning experiences: Practical implications and outcomes", Computers \& Education, vol. 63, no. 0, (2013).

[8] G. Zichermann and C. Cunningham, "Gamification by Design: Implementing Game Mechanics in Web and Mobile Apps", Q'Reilly Media, (2011).

[9] Energy Efficiency Exchange, Heating, Ventilation and Air Conditioning [Report]. Available: http://eex.gov.au/technologies/heating-ventilation-and-air-conditioning/\#fn-190-1, (2013) November.

[10] N. Wang, J. Zhang and X. Xia, "Energy consumption of air conditioners at different temperature set points", Energy and Buildings, vol. 65, no. 0, (2013).

[11 H. Sachs, A. Lowenberger and W. Lin, "Emerging Energy-Saving HVAC Technologies and Practices for the Bulldings Sector", [Research Report A092]. Available: http://www.aceee.org/research-report/a092, (2009) November.

[12] Y. J. Huang, H. Akbari, H. Taha and A. H. Rosenfeld, "The potential of vegetation in reducing summer cooling loads in residential buildings", Journal of Climate and Applied Meteorology, vol. 26, no. 9, (1987).

[13] D. B. Crawley, J. W. Hand, M. Kummert and B. T. Griffith, "Contrasting the capabilities of building energy performance simulation programs", Building and Environment, vol. 43, no. 4, (2008).

[14] J. Hirsch, "Quick Energy Simulation Tool (eQUEST): Introductory tutorial", [Web resource]. Available: http://doe2.com/download/equest/eQ-v3-64_Introductory-Tutorial.pdf.

[15] Aloha Systems Inc, "Evaluation, measurement, and verification final report", CALifornia Measurement Advisory Council (CALMAC), (2006) May 19. 
[16] Simulation Research Group Lawrence Berkley National Lab. (2009, August). Overview of DOE 2.2 [WWW page]. Available: http://doe2.com/.

[17] Lawrence Berkeley National Laboratory, "Getting Started with EnergyPlus", [Web resource]. Available: http://apps1.eere.energy.gov/buildings/energyplus/, (2011) June.

[18] B. Fogg, "Persuasive Technology: Using Computers to Change What We Think and Do", Morgan Kaufmann, San Francisco, CA, USA, (2003).

[19] A. Sears and J. A. Jacko, "Human-Computer Interaction Fundamentals", CRC Press, Taylor and Francis Group, Boca Raton, FL, USA, (2009).

[20] A. MacLean, K. Carter, L. Lövstrand and T. Moran, "User-tailorable systems: pressing the issues with buttons", Presented at the Proceedings of the SIGCHI Conference on Human Factors in Computing Systems, Seattle, Washington, United States, (1990).

[21] S. Consolvo, D. W. McDonald and J. A. Landay, "Theory-driven design strategies for technologies that support behavior change in everyday life", Presented at the Proceedings of the SIGCHI Conference on Human Factors in Computing Systems (CHI 09), Boston, MA, USA, (2009).

[22] R. Oppermann, "User-interface Design", Handbook on Information Technologies for Education and Training. H. Adelsberger, B. Collis, and J. Pawlowski, Eds., first ed Springer Berlin Heidelberg Gemany (2002), pp. 233-248.

[23] W. O. Galitz, "The essential guide to user interface design: an introduction to GUI design principles and techniques", Wiley Publishing Inc., Indianapolis, Indianam USA, (2007)

[24] M. Alavi and D. E. Leidner, "Knowledge management systems. ssues, challenges, and benefits", Communications of the AIS. 1, 2es, (1999).

[25] Unity Technologies, "The Unity game development engine", WWWW site]. Available: http://unity3d.com/, (2013).

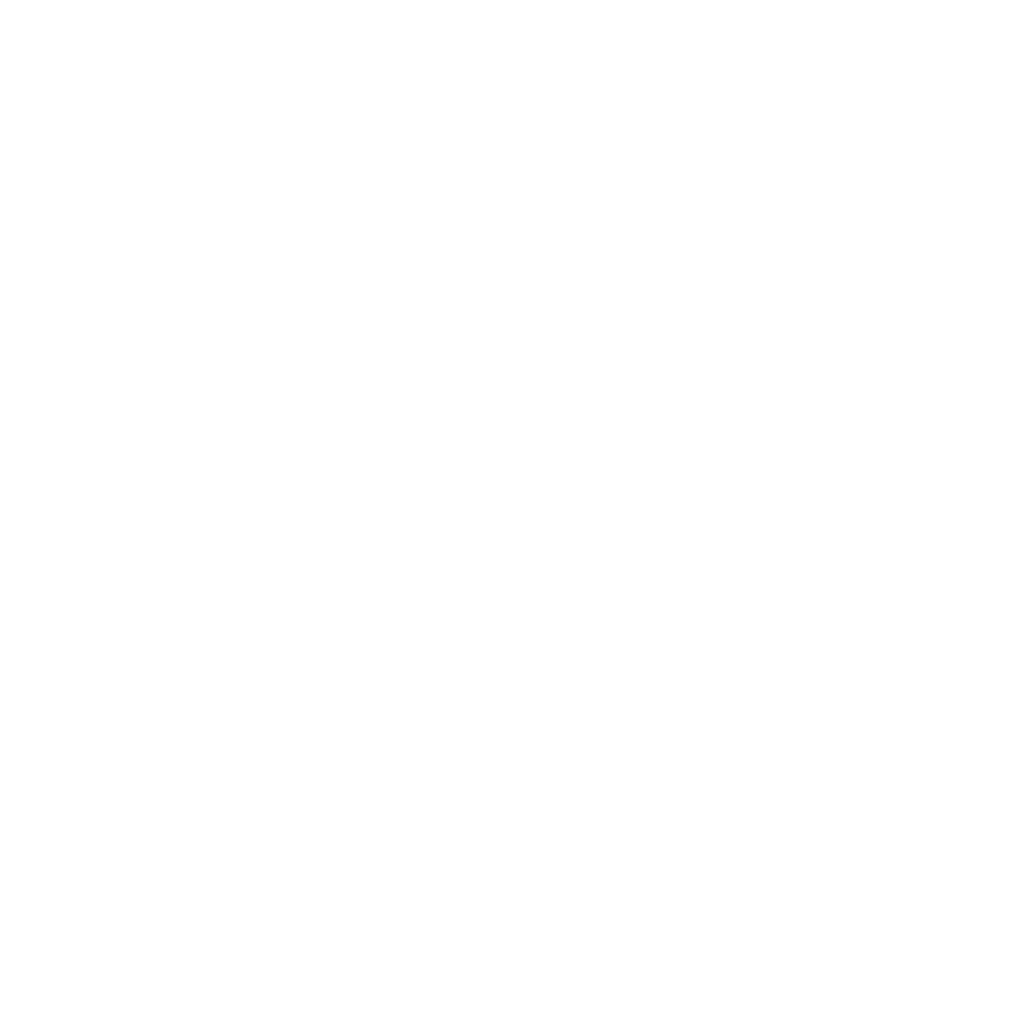

